SHS Web of Conferences 10, 00030 (2014)

DOI: $10.1051 /$ shsconf/20141000030

C Owned by the authors, published by EDP Sciences, 2014

\title{
The relationship between children's reading ability, verbal and fluid intelligence and measurements of eye movements during reading
}

\author{
M. Orlovska, K. Bluss, and M. Rascevska \\ University of Latvia, Latvia
}

\begin{abstract}
The aim of this study was to clarify, which of the following two measures: verbal intelligence or measurements of eye movements, is better predictor of reading ability. In addition, the study also investigated the relationships between reading ability, fluid intelligence and measurements of eye movements. Participants of the study $(\mathrm{N}=28$; mean age $=8.80 ; \mathrm{SD}=.41 ; 54 \%$ boys) were assessed in reading with LMST-I Reading achievement test, verbal and fluid intelligence was measured using two scales - Verbal Comprehension and Perceptual reasoning - from Latvian edition of Wechsler Intelligence Scale for Children Fourth Edition (WISC- IV, Latvian version), as well as eye tracking was made during reading. The results show that reading ability is better predicted by fixation duration and fixation count measurements of eye movements for 8-9 old children, whereas verbal ability in general does not predict reading ability. The better link with level of reading is provided by measurements of eye movements, but not so accurately reflected by verbal ability in appropriate age group, when the acquisition of reading ability still continues. No relationships among children's fluid intelligence, reading ability and measurements of eye movements were reported.
\end{abstract}

\section{Introduction}

Reading ability is very important in self-directed process of learning; therefore, early diagnostic of reading difficulties, which could be provided with effective assessment tools of reading ability and related cognitive abilities, is relevant.

Reading ability includes lower and higher level processes. The lower-level processes refer to such cognitive components as ability to visually percept letters and symbols and to decode them, differentiate sounds and integrate them into words, while higher-level processes consist of ability to comprehend, memorize and integrate information accurately, creating a deeper understanding and causality about the given text (Van der Schoot, Vasbinder, Horsley, \& Van Lieshout, 2008; Tilstra, Van den Broek, Kendeou, \& Rapp, 2009).

According to Bootleneck hypothesis lower-lever processes play more significant role at the beginning of reading acquisition (Perfetti, 1985). The importance of memory, comprehension and formation of causal relationships, on the other hand, increases, when basic reading skills are acquired in sufficiently automatic level. Since processes of the both level may operate in a different way, it is possible to observe individual differences in reading ability. For example, children with specific kind of

This is an Open Access article distributed under the terms of the Creative Commons Attribution License 4.0, which permits unrestricted use, distribution, and reproduction in any medium, provided the original work is properly cited. 


\section{SHS Web of Conferences}

reading disability - dyslexia, often have poor phonological ability and deficit of visual processing and more infrequently also limited motion processing (Fiorello, Hale, \& Snyder, 2006). However, sometimes such individuals may show peculiar talent in the visual-spatial area (Winner et al, 2001; Attree, Turner, \& Cowell, 2009). Differences also may be determined by type of a language, for example, when reading in a language with transparent orthography (like Latvian language), understanding of grapheme-phoneme correspondence is acquired much faster than in languages with more opaque orthography, such as English (Jimenez, Siegel, \& Lopez, 2003).

Theory shows that reading ability is related to general intelligence and, hence, intelligence may predict reading ability (Glutting, Watkins, Konold, \& McDermott, 2006). Alternative approach in predicting reading ability is to use only verbal intelligence as it is highly related to general intelligence (Carrol, 1993) and well predicts reading (Stage, Abbott, Jenkins, \& Berninger, 2003). There is no clear and consequent evidence about the relationships between reading ability and fluid intelligence (Jimenez, Siegel, \& Lopez, 2003), thus these relationships have to be investigated more.

Eye tracking is a widely used approach which reflects the process going on during reading. In the past few decades it has been extensively used for studies of reading in languages like English, Spanish and other (Rayner, 1998), as well as applied to studies of reading in Latvian language (Paeglis, 2010). The findings reveal that reading ability is related to several parameters of eye movements. For example, more proficient readers show shorter fixation durations, longer saccades and less regressions than novice readers (Reichle, Rayner, \& Pollatsek, 2003; Paeglis, Gorshanova, Bagucka, \& Lacis, 2008; Paeglis, Bagucka, Gorshanova, \& Balgalve, 2009). Through eye tracking we may observe that, in general, the process of reading for less proficient readers, compared to more proficient ones, is qualitatively similar, only with lower efficacy. Children compared to adults show considerably longer fixation durations, thus reflecting that for children who still have not acquired reading ability in sufficiently automatic level higher fixation durations indicate the longer process of syllable or word processing (Paeglis, Orlovska, \& Bluss, 2011).

Overall intelligence and measurements of eye movements play a very important role in prediction of reading ability. For better understanding of reading ability and further implications for reading disability diagnosis and intervention, our study investigated the following questions: Which is better predictor of reading ability - verbal intelligence or measurements of eye movements during reading? In addition, we wanted to find out: What is the relationship between reading ability, fluid intelligence and measurements of eye movements during reading? Is the measure of reading of the text during eye tracking related to the LMST-I measurement of reading of the text and confirms their reciprocal convergent validity?

\section{Methods}

\section{Participants}

Thirty one participant - Latvian speaking pupils of the $2^{\text {nd }}$ grade aged $8-9$ took part in the study. Three participants were excluded from further data analysis because of the lack of measurements of eye movements or because of not fitting in $2^{\text {nd }}$ grade age group. Analysis was done for data of 28 participants (mean age $=8.80$ years; $\mathrm{SD}=.41$ ). Sample included $54 \%$ boys. Participants were from several mainstream schools without a specialization in a particular school subject. All children had written permission from their parents that confirmed parent agreement for child's participation in the study.

\section{Measures}

Assessment of reading ability was done with recently developed LMST-I Reading achievement test (RAT) (Raščevska, Paegle, \& Mencis, 2010). Measurement on RAT takes into account both - reading 


\section{Int. Conf. SOCIETY. HEALTH. WELFARE.}

speed and accuracy. The adapted version in Latvia of Wechsler Intelligence Scale for Children Fourth Edition (WISC-IV) (Wechsler, 2003; adaptation in Latvia by Raščevska \& Sebre, 2008) was used to measure verbal and fluid intelligence. Subtests of Verbal comprehension scale (Similarities, Vocabulary and Comprehension) refer to assessment of verbal intelligence, while subtests of Perceptual reasoning scale (Picture Concepts, Matrix Reasoning and Block Design) refer to assessment of fluid intelligence. Measurements of eye movements were recorded with Eye tracking device (SensoMotoric Instruments (SMI), 2009). The device is used for the stimuli of a confined area, such as page screenshot. The setup calculates observer's gaze direction from the infrared video of the eye pupil area, it compensates for the head movements by tracking the corneal reflection. The data-sampling rate of this device is $250 \mathrm{~Hz}$; the tracking resolution is less than $0.01 \mathrm{deg}$ and gaze position accuracy $0.5 \mathrm{deg}$. Participants were sitting in a free position $80 \mathrm{~cm}$ from a 22-inch flat panel monitor. This monitor was plugged into the iViewX laptop (IBM) that displayed different elements, such as texts, on both screens. During eye tracking we used two specially created texts for reading and analyzed two parameters of eye movements - fixation duration and fixation count.

\section{Procedure}

Testing was done during two or three individual sessions with each participant. In the first session child had assessment of reading, which took about 5-10 minutes, then assessment of intelligence, about 30-45 minutes long. On the second session eye tracking was done, which took about 10 minutes. During eye tracking, first, each child had a calibration procedure to adjust device to particular child's peculiarity of a gaze. After calibration "practise" text appeared on the screen. Participant had to read it loudly, and parameters of eye movements obtained during this reading were not selected for the analysis. When child had finished reading the "practise" text, the main text appeared, and again - child had to read it loudly, but this time parameters of eye movements, such as fixation duration and fixation count, were included in data analysis.

\section{Data analysis}

The software by SensoMotoric Instruments collected eye movement data. We did initial data processing by BeGaze 2.3 (SensoMotoric Instruments), then by IBM SPSS Statistics 19.0.

\section{Results}

Table 1 summarizes parameters of descriptive statistics for data obtained in the study.

WNL - Word non-word reading ; TR - Text reading; BD - Block design; SI - Similarities; PCPicture concepts; VO - Vocabulary; MR - Matrix reasoning; CO - Comprehension; Fix.dur.(ms) Fixation duration in miliseconds; Fix. count - Fixation count.

Spearmen-Brown split-half reliability is acceptable for all measures ( $\mathrm{rsb}>=.70)$ (see Table 1).

Pearson correlation coefficient indicates statistically significant medium strong $(\mathrm{r}=0.46 ; \mathrm{p}<.05)$ relationship between Text reading subtest of LMST-I and Vocabulary subtest of WISC - IV. The medium strong, significant correlation $(\mathrm{r}=-.50 ; \mathrm{p}<.01)$ was reported between fixation duration and subtest of Text reading. The opposite medium strong significant relationship $(\mathrm{r}=-.46 ; \mathrm{p}<.05)$ between fixation duration and the results in the subtest of Picture concepts was found. No significant correlations between subtest of Text reading and subtest of Similarities $\left(\mathrm{r}=.31, \mathrm{p}>.05 ; \mathrm{r}_{s}=.30, \mathrm{p}>.05\right)$, the subtest of Text reading and the subtest of Comprehension $\left(\mathrm{r}=.28, \mathrm{p}>.05 ; \mathrm{r}_{s}=.32, \mathrm{p}>.05\right)$ were reported.

We found no significant relationship between subtest of Word non-word reading and fixation duration $(\mathrm{r}=-.18 ; \mathrm{p}>.05)$, nor between fixation count and subtest of Word non-word reading $\left(\mathrm{r}=-.32, \mathrm{p}>.05 ; \mathrm{r}_{\mathrm{s}}=-.23, \mathrm{p}>.05\right)$, nor between subtest of Word non-word reading and any 


\section{SHS Web of Conferences}

Table 1. Parameters of descriptive statistics of LMST-I Reading achievement test, WISC-IV Perceptual reasoning and Verbal comprehension scale subtests and measurements of eye movements (all raw scores) for 8-9 year old pupils.

\begin{tabular}{|c|c|c|c|}
\hline Variables & M & SD & $\begin{array}{c}\text { Spearmen-Brown } \\
\text { coefficient }\end{array}$ \\
\hline \multicolumn{4}{|c|}{ Scales of LMST-I Reading achievement test } \\
\hline WNR & 26.57 & 9.53 & .94 \\
\hline TR & 66.09 & 23.83 & .99 \\
\hline \multicolumn{4}{|c|}{ Scales of WISC-IV } \\
\hline BD & 29.29 & 9.35 & .90 \\
\hline SI & 15.43 & 7.47 & .94 \\
\hline $\mathrm{PC}$ & 15.89 & 3.76 & .70 \\
\hline $\mathrm{VO}$ & 27.21 & 10.77 & .93 \\
\hline MR & 16.39 & 4.94 & .96 \\
\hline $\mathrm{CO}$ & 17.04 & 4.53 & .88 \\
\hline \multicolumn{4}{|c|}{ Measurements of eye movements } \\
\hline Fix dur. (ms) & 325 & 98 & - \\
\hline Fix. count & 183 & 92 & - \\
\hline
\end{tabular}

of WISC-IV subtests. There were no significant correlations also between the subtests of Perceptual reasoning scale and subtest of Text reading and Word non-word reading.

Significant high positive correlation was found between LMST-I measure of text reading and measure of text reading during eye tracking task $(\mathrm{r}=.83 ; \mathrm{p}<.0001)$, thus, confirming reciprocal convergent validity of the both measurements.

To find out how variables are connected to each other in the level of scales and which of them better predicts reading ability, raw scores of subtests of RAT were transformed into standardized scores $(\mathrm{M}=100 ; \mathrm{SD}=15)$ within the sample and combined in one measure that applies to reading ability. The raw scores of WISC-IV subtests $\mathrm{BD}, \mathrm{PC}$ and $\mathrm{MR}$ were transformed into standardizes scores $(\mathrm{M}=100$; $\mathrm{SD}=15$ ) within the sample and combined in one measure - Perceptual reasoning that applies to fluid intelligence. The raw scores of WISC-IV subtests SI, VO and CO were transformed into standardized scores $(M=100 ; S D=15)$ and combined in one measure - Verbal comprehension and applies to verbal intelligence. The raw scores of fixation duration and count were transformed into standardized scores $(\mathrm{M}=100 ; \mathrm{SD}=15)$.

Pearson correlation coefficient showed the only significant relationships important in the context of research between reading ability and fixation duration $(\mathrm{r}=-.41 ; \mathrm{p}<.05)$, and between reading ability and fixation count $(\mathrm{r}=-.38 ; \mathrm{p}<.05)$.

To find out what better predicts reading ability, first of all, hierarchical regression model was created in the level of separate cognitive tasks (see Table 2).

According to hierarchical regression analysis with dependent variable performance in subtest of Text reading, in the first step independent variable fixation duration explains $25 \%$ of dependent variable $\left(\mathrm{R}^{2}=.25 ; \mathrm{F}(1,26)=8.48 ; \mathrm{p}<.01\right)$. In the second step independent variables fixation duration and performance in subtest of Vocabulary together explain $38 \%$ of dependent variable $\left(\mathrm{R}^{2}=.38 ; \mathrm{F}(2,25)=\right.$ $7.71 ; \mathrm{p}<.01)$.

Table 3 shows hierarchical regression model for dependent variable reading ability and independent variables fixation duration and fixation count.

According to hierarchical regression analysis, in the first step independent variable fixation duration explains $17 \%$ or reading ability $\left(\mathrm{R}^{2}=.17 ; \mathrm{F}(1,26)=5.21 ; \mathrm{p}<.05\right)$. In the second fixation duration and fixation count together explain $39 \%$ of reading ability $\left(\mathrm{R}^{2}=.39 ; \mathrm{F}(2,25)=8.06 ; \mathrm{p}<.01\right)$. 
Int. Conf. SOCIETY. HEALTH. WELFARE.

Table 2. Hierarchical regression analysis (with raw scores) for dependent variable performance in subtest of Text reading and independent variables fixations duration and performance in Vocabulary subtest of WISC-IV.

\begin{tabular}{lccc}
\hline Independent variable & B & SE B & $\beta$ \\
\hline 1. step & & & \\
Fixation duration & -.12 & .04 & $-.50^{* *}$ \\
\hline 2. step & & & \\
Fixation duration & -.10 & .04 & $-.43^{* *}$ \\
$\quad$ Vocabulary subtest & .83 & .35 & $.38^{*}$ \\
\hline $\mathrm{p}<.05, * * \mathrm{p}<.01$ & & &
\end{tabular}

Table 3. Hierarchical regression analysis (with standardized scores) for dependent variable reading ability and independent variable fixation duration and fixation count.

\begin{tabular}{llll}
\hline Independent variable & B & SE B & $\beta$ \\
\hline 1. step & & & \\
$\quad$ Fixation duration & -91.89 & 40.28 & $-.41^{*}$ \\
2. step & & & \\
$\quad$ Fixation duration & -114.58 & 35.87 & $-.51^{* *}$ \\
Fixation count & -109.15 & 35.87 & $-.49^{* *}$ \\
$* \mathrm{p}<.05, * * \mathrm{p}<.01$ & & &
\end{tabular}

\section{Discussion}

The main aim of this study was to clarify, whether verbal intelligence or measurements of eye movements is the better predictor of reading ability. Our data report that reading ability for 8-9 year old children is predicted by fixation duration and fixation count parameters of eye movements, but is not predicted by verbal intelligence. The answer on the second research question about relationship between reading ability, fluid intelligence and measurements of eye movements, is that reading ability for 8-9 year old children is not related to fluid intelligence, in its turn, fluid intelligence represented by Picture concepts subtest of WISC-IV is related to fixation duration, but fluid intelligence in general is not related to fixation count.

According to the results children, who read the given text more fluently and accurately have deeper comprehension of the concepts and shorter fixation duration. The findings are supported by the previous studies, which report that children with better reading ability can better explain meanings of the words (Jimenez, Siegel, \& Lopez, 2003; Cain \& Oakhill, 2006), and children having reading difficulties make longer fixations while processing text (Rayner, 1998; Reichle, Rayner, \& Pollatsek, 2003). Fixation duration together with comprehension of the concepts to great extent (39\%) explain reading of 8-9 year old pupils, thus, such combination of cognitive tasks may be successfully applied to reading ability explanation in particular age group.

Although previously it has been advocated that individuals with reading disability may be talented in visual-spatial area (Attree, Turner, \& Cowell, 2009), or show low abilities of fluid reasoning (Winner et al. 2001), in the present study the reading of 8-9 year old children is not related to fluid intelligence. Also the fixation duration and fixation count are not related to perceptual reasoning in general. However, in the level of cognitive tasks which require ability of fluid reasoning, we observe that children, who have better abstract reasoning, have shorter fixation duration while processing a text. This is supported by the study about eye movements during analogy solving, where those individuals, who showed longer fixation durations processing every item, had weaker ability of fluid reasoning (Vakil, Lifshitz, Tzuriel, Weiss, \& Arzuoan, 2011).

The reading speed of text reading during eye tracking is strongly correlated to reading speed and reading accuracy measures in text reading of LMST-I and that confirms reciprocal convergent validity of the measures. 


\section{SHS Web of Conferences}

The limitations of the study include quite small sample size and inclusion of the participants by availability principle rather than randomly.

\section{Conclusions}

The present study shows that reading ability for 8-9 year old children is highly predicted by fixation duration and fixation count of eye movements, but not predicted by verbal intelligence. Fluid intelligence of the children is not related to reading ability, nor to measurements of eye movements. The better readers have deeper comprehension of concepts, while those with better abstract reasoning can faster process visual information and their fixation durations are shorter.

Measure of text reading in Reading achievement test is strongly correlated with text measure obtained during eye tracking and confirms reciprocal convergent validity of the measures.

\section{References}

[1] Attree, E., Turner, M. J., \& Cowell, N. (2009). A virtual reality test identifies the visuospatial strengths of adolescents with dyslexia. Cyberpsychology \& Behavior, 12 (2), 163-168

[2] Cain, K., \& Oakhill, J. (2006). Profiles of children with specific reading comprehension difficulties. British Journal of Educational Psychology, 76, 683-696

[3] Carroll, J. B. (1993). Human cognitive abilities: A survey of factor-analytical studies. New York: Cambridge University Press

[4] Fiorello, C. A., Hale, J. B., \& Snyder, L. E. (2006). Cognitive hypothesis testing and response to intervention for children with reading problems. Psychology in the Schools, 43, 835-853

[5] Glutting, J. J., Watkins, M. W., Konold, T. R., \& McDermott, P. A. (2006). Distinctions without a difference. The Journal of Special Education, 40 (2), 103-114

[6] Jimenez, J. E., Siegel, L. S., \& Lopez, M. R. (2003). The Relationship between IQ and reading disabilities in English-speaking Canadian and Spanish children. Journal of Learning Disabilities, $36(1), 15-23$

[7] Linderholm, T., Everson, M. G., Van der Broek, P., Mischinski, M., Crittenden, A., \& Samuels, J. (2000). Effects of causal text revisions on more - and less-skilled readers' comprehension of easy and difficult texts. Cognition and Instruction, 18 (4), 525-556

[8] Paeglis, R. (2010). Movements of eyes in real tasks. Berlin: Lambert Academic Publishing.

[9] Paeglis, R., Gorshanova, I., Bagucka K., \& Lacis, I. (2008). Latvian and Russian textbooks: eye movements in reading text formatted in two columns. In J. Spigulis, A. Krumins, D. Millers, A. Sternberg, I. Muzikante, A. Ozols, M. Ozolinsh (Eds.). Sixth International Conference on Advanced Optical Materials and Devices (AOMD-6), Riga, Latvia. Proceedings of SPIE, 7142, 19

[10] Paeglis, R., Bagucka, K., Gorshanova, I., \& Balgalve, A. (2009). Eye movement patterns and reading speed in two highly inflected languages written with different alphabets. Latvian Journal of Physics and Technical Sciences, 4, 15-22

[11] Paeglis, R., Orlovska, M., \& Bluss, Kr. (2011). Color stabilizes textbook visual processing. In F. Baldini, J. Homola, R. A. Lieberman, \& K. Kalli (Eds.). Optical sensors 2011; and photonic crystal fibers V, Prague, Czech Republic. Proceedings of SPIE, 8073, 16

[12] Perfetti, C. A. (1985). Reading ability. New York: Oxford University Press

[13] Raščevska M., \& Sebre S. (2008). Latvian edition of Wechsler Intelligence Scale for Children. Univeristy of Latvia, EPF Department of Psychology, unpublished material

[14] Raščevska, M., Paegle, Dz., \& Mencis, J. (2010). Assessments manual of Latvian language and maths achievement test. Riga: LU Academic publishing 
Int. Conf. SOCIETY. HEALTH. WELFARE.

[15] Rayner, K. (1998). Eye movements in reading and information processing: 20 years of research. Psychological Bulletin, 124 (3), 372-422

[16] Reichle, E. D., Rayner, K., \& Pollatsek, A. (2003). The E-Z Reader model of eye movement control in reading: Comparisons to other models. Behavioral and Brain Sciences, 26, 445-476

[17] Stage, S. A., Abbott, R. D., Jenkins, J. R., \& Berninger, V. W. (2003). Predicting response to early reading intervention from verbal IQ, reading-related language abilities, attention ratings, and verbal IQ-word reading discrepancy. Journal of Learning Disabilities, 36, 24-33

[18] Tilstra, J., Van den Broek, P., Kendeou, P., \& Rapp, D. (2009). Simple but complex: Components of the simple view of reading across grade levels. Journal of Research in Reading, 32 (4), 382-401

[19] Vakil, E., Lifshitz, H., Tzuriel, D., Weiss, I., \& Arzuoan, Y. (2011). Analogies solving by individuals with and without intellectual disability. Research in Developmental Disabilities, 32 (2), 846-856

[20] Van der Schoot, M., Vasbinder, A. L., Horsley, T. M., \& Van Lieshout, E. C. D. M. (2008). The role of two reading strategies in text comprehension. Journal of Research in Reading, 31 (2), 203 223

[21] Wechsler, D. (2003). Wechsler Intelligence Scale for Children-Fourth Edition. San Antonio, TX: Psychological Corporation

[22] Winner, E., Von Karolyi, C., Malinsky, D., French, L., Seliger, C., Ross, E., \& Weber, C. (2001). Dyslexia and visual-spatial talents compensation vs deficit model. Brain \& Language, 76, 81-110 